\title{
MicroRNA-608 sensitizes non-small cell lung cancer cells to cisplatin by targeting TEAD2
}

\author{
YANLI WANG $^{1}$, FENGCAI LI $^{1}$, DANDAN MA $^{1}$, YUHUA GAO $^{1}$, RUNPU LI $^{1}$ and YINGJIE GAO $^{2}$ \\ Departments of ${ }^{1}$ Oncology and ${ }^{2}$ Hematology, Baoding No. 2 Central Hospital, Zhuozhou, Hebei 072750, P.R. China
}

Received September 27, 2018; Accepted April 3, 2019

DOI: $10.3892 / \mathrm{mmr} .2019 .10616$

\begin{abstract}
Cisplatin has been widely used as a conventional treatment for patients with non-small cell lung cancer (NSCLC). However, primary and acquired cisplatin resistances are frequently developed during the treatment of patients with NSCLC, leading to an increased mortality rate. Accumulating evidence demonstrated that aberrantly expressed microRNAs (miRs) are involved in the development of chemoresistance. In the present study, sensitivity of NSCLC cells to cisplatin was identified to increase following overexpression of miR-608. Conversely, sensitivity to cisplatin was reduced following miR-608 knockdown. Reverse transcription-quantitative PCR and western blotting analyses identified that TEA domain transcription factor 2 (TEAD2), a key regulator of cell stemness, was negatively regulated by miR-608 in NSCLC cells. By repressing TEAD2, miR-608 decreased the expression level of several target genes of the Hippo-yes-associated protein signaling pathway. Furthermore, TEAD2 mRNA was confirmed to be targeted by miR-608 in NSCLC cells via a dual-luciferase reporter assay. Importantly, the increased cisplatin sensitivity induced by miR-608 overexpression was reversed by transfection of TEAD2 in NSCLC cells. The present data suggested that miR-608 may represent a novel candidate biomarker for the evaluation of cisplatin sensitivity in patients with NSCLC.
\end{abstract}

\section{Introduction}

Lung cancer is the most common malignancy worldwide, with $\sim 1.8$ million patients diagnosed and 1.3 million cancer-related mortalities every year (1). Non-small cell lung cancer (NSCLC) is the most aggressive form of lung cancer (2). Chemotherapy has been widely used for the treatment of patients with NSCLC (3). Despite positive response at the initial stages of the treatment, development of chemoresistance may cause

Correspondence to: Dr Yingjie Gao, Department of Hematology, Baoding No. 2 Central Hospital, 57 Fanyang Middle Road, Zhuozhou, Hebei 072750, P.R. China

E-mail: gaoyingjiebd@sohu.com

Key words: microRNA-608, non-small cell lung cancer, cisplatin failure of cancer therapy, representing a major challenge for the treatment of patients with NSCLC (4). Therefore, understanding the molecular mechanism of NSCLC is required to develop novel strategies to overcome chemoresistance.

MicroRNAs (miRNAs/miRs) are a group of small, non-coding, single-stranded RNA molecules that can negatively regulate gene expression by directly binding to the 3' untranslated region (3'UTR) of their target mRNAs (5). miRNAs play key roles in regulating various physiological cellular processes, such as cell differentiation, cell motility and cell apoptosis (6). Dysregulation of miRNAs is an important mechanism underlying the development of various types of cancer, including NSCLC (7). Additionally, numerous previous studies identified multiple miRNAs involved in the development of chemoresistance (8-10). Downregulation of miR-138 increases the expression of zinc finger E-box binding homeobox 2 , and induces epithelial-mesenchymal transition (EMT) in NSCLC cells, thus leading to adriamycin resistance (11). Single-nucleotide polymorphisms of miR-608, a recently identified miRNA, were found in several types of cancer $(12,13)$. A previous study demonstrated that miR-608 regulates apoptosis of A549 cells (14). However, the role of miR-608 in the development of chemoresistance remains unknown.

In mammals, the TEA domain transcription factors (TEAD) family consists of four members (15). As a transcription factor, TEAD2 regulates the expression of numerous genes and it is involved in various physiological processes (16-18). The activity of TEAD2 depends on the physical interaction with its transcription co-activator yes-associated protein (YAP) and transcriptional coactivator with PDZ-binding motif (TAZ), which are regulated by the Hippo tumor suppressor signaling pathway (19). During the development of cancer, the Hippo signaling pathway may be impaired, leading to the overactivation of the YAP/TAZ-TEAD2 complex $(19,20)$. In breast cancer cells, elevated activity of the YAP/TAZ-TEAD2 complex promotes cancer cell growth and metastasis (21). In murine normal and cancer cells, overexpression of TEAD2 leads to the nuclear accumulation of YAP/TAZ, causing metastasis (22). Although several previous studies have demonstrated the pivotal role of TEAD2 in cancer progression, the mechanism of TEAD2 in cancer remains unclear.

In the present study, cell viability and apoptosis assays demonstrated that miR-608 regulates cisplatin sensitivity in A549 cells. Furthermore, TEAD2 expression was negatively 
regulated by miR-608 in A549 cells. By repressing the expression level of TEAD2, miR-608 caused a decreased in the expression level of several genes downstream of Hippo-YAP signaling. In addition, TEAD2 was identified to be a direct target of miR-608 in A549 cells via a dual-luciferase reporter assay. Importantly, cell viability and apoptosis assays suggested that the increased cisplatin sensitivity induced by miR-608 was reversed by TEAD2 overexpression in A549 cells. The present results suggested that miR-608 may represent a novel candidate biomarker for the evaluation of cisplatin sensitivity in patients with NSCLC.

\section{Materials and methods}

Cell culture and reagent. The human NSCLC cell line A549 was purchased from The American Type Culture Collection. The A549 cells were cultured in DMEM (Gibco; Thermo Fisher Scientific, Inc.) supplemented with 10\% FBS (HyClone; GE Healthcare Life Sciences) and 1\% penicillin-streptomycin (Invitrogen; Thermo Fisher Scientific, Inc.) in a humidified incubator at $37^{\circ} \mathrm{C}$ with $5 \% \mathrm{CO}_{2}$. Cisplatin was purchased from Selleck Chemicals and dissolved in DMSO to a concentration of $10 \mathrm{mM}$. For the cell viability and apoptosis assays, cisplatin was diluted in DMSO to a final concentration of 5, 10, 15 and $20 \mu \mathrm{M}$.

Overexpression and inhibition of $m i R-608$. miR-NC mimic, miR-608 mimic, miR-NC inhibitor and miR-608 inhibitor were synthesized and purchased from GenePharma. To overexpress or silence miR-608, miR-608 mimic or miR-608 inhibitor were transfected into A549 cells using Lipofectamine ${ }^{\circledR} 3000$ (Invitrogen; Thermo Fisher Scientific, Inc.) according to the manufacturer's protocol. In total, $50 \mathrm{nM}$ miR-608 mimic or miR-608 inhibitor were incubated with Lipofectamine ${ }^{\circledR} 3000$ reagent in serum-free DMEM for $15 \mathrm{~min}$. The mixture was added to cells $\left(2 \times 10^{3} /\right.$ well) cultured in 96 -well plates and incubated for 3 days prior to RNA or protein extraction. The sequences used in the transfection experiments were as follows: miR-NC mimic, 5'-UCGCUUGGUGCAGGUCGG GAA-3'; miR-608 mimic, 5'-AGGGGUGGUGUUGGGACA GCUCCGU-3'; miR-NC inhibitor, 5'-UUCUCCGAACGU GUCACGUTT-3'; miR-608 inhibitor, 5'-ACGGAGCUGUCC CAACACCACCCCU-3'.

Cell viability assay. To determine the cytotoxicity of cisplatin in A549 cells, the cell viability was detected using a Cell Counting Kit-8 (CCK-8; Dojindo Molecular Technologies, Inc.) according to the manufacturer's protocol. In total, $2 \times 10^{3}$ cells/well were seeded in 96-well plates and incubated overnight in an incubator at $37^{\circ} \mathrm{C}$ with $5 \% \mathrm{CO}_{2}$. On the following day, cells were transfected with miR-608 mimic, miR-608 inhibitor, miR-negative control (NC) mimic or miR-NC inhibitor; 2 days later, cells were treated with increasing concentrations of cisplatin or DMSO. After 3 days, $10 \mu 1$ CCK-8 solution was added into each well in 96-well plates and cells were incubated at $37^{\circ} \mathrm{C}$ for $2 \mathrm{~h}$. The medium containing CCK-8 was transferred to a 96 -well plate. The absorbance in each well was measured at $450 \mathrm{~nm}$ using a microplate reader. To investigate the effects of miR-608 on cell viability, untreated cells were examined 3 days after transfection with
miR-608 mimic, miR-608 inhibitor, miR-negative control (NC) mimic or miR-NC inhibitor.

Cell apoptosis assay. The percentage of apoptotic cells was analyzed using an Annexin V-FITC apoptosis detection kit (Sigma-Aldrich; Merck KGaA) according to the manufacturer's protocol. After transfection or drug treatment, cells were collected and resuspended, and $1 \times 10^{6}$ cells $/ \mathrm{ml}$ were incubated with $100 \mu \mathrm{l} 1 \mathrm{X}$ binding buffer. Subsequently, $2 \mu 1$ Annexin V-FITC was added into the mixture and incubated at room temperature for $15 \mathrm{~min}$. Then, $400 \mu \mathrm{l}$ PBS and $1 \mu 1$ propidium iodide were added and incubated for $5 \mathrm{~min}$ at room temperature. The cells were analyzed using a FACSCalibur flow cytometer (BD Biosciences) and the data were analyzed using the FlowJo software (version 10.2; FlowJo LLC).

$R N A$ extraction and reverse transcription-quantitative $P C R$ $(R T-q P C R)$. Total RNA was extracted from A549 cells using TRIzol reagent (Invitrogen; Thermo Fisher Scientific, Inc.) according to the manufacturer's protocol. The stem-loop method was used to detect miR-608 expression. RNA was reverse transcribed into cDNA using TransScript First-Strand cDNA Synthesis SuperMix (Beijing TransGen Biotech Co., Ltd.) at $65^{\circ} \mathrm{C}$ for $5 \mathrm{~min}$, followed by incubation in ice for 2 min. The qPCR was performed using SYBR Premix Ex Taq (Takara Bio, Inc.) with a CFX-96 Realtime PCR System (Bio-Rad Laboratories, Inc.). The thermocycling conditions were as follows: Initial denaturation at $95^{\circ} \mathrm{C}$ for $30 \mathrm{sec}$, followed by 40 cycles of $95^{\circ} \mathrm{C}$ for $5 \mathrm{sec}$ and $60^{\circ} \mathrm{C}$ for $30 \mathrm{sec}$. Using the $2^{-\Delta \Delta \mathrm{Cq}}$ method (23), U6 and $\beta$-actin were used as internal controls for calculating the relative expression levels of miRNA and mRNAs, respectively. The primer sequences used were as follows: miR-608 stem-loop primer, 5'-CTCAACTGG TGTCGTGGAGTCGGCAATTCAGTTGAGACGGAG-3'; miR-608, forward 5'-GCCGAGTCGCTTGGTGCAGG-3' and reverse 5'-CTCAACTGGTGGTGTCGTGGA-3'; U6, forward 5'-TGCGGGTGCTCGCTTCGCAGC-3' and reverse 5'-CCA GTGCAGGGTCCGAGGT-3'; TEAD2, forward 5'-CTTCGT GGAACCGCCAGAT-3' and reverse 5'-GGAGGCCACCCT TTTTCTCA-3'; connective tissue growth factor (CTGF), forward 5'-CAGCATGGACGTTCGTCTG-3' and reverse 5'-AACCACGGTTTGGTCCTTGG-3'; cysteine-rich angiogenic inducer 61 (CYR61), forward 5'-CTCGCCTTAGTC GTCACCC-3' and reverse 5'-CGCCGAAGTTGCATTCCA G-3'; inhibin subunit $\beta$ A (Inhba), forward 5'-CCTCCCAAA GGATGTACCCAA-3' and reverse 5'-CTCTATCTCCACATA CCCGTTCT-3'; $\beta$-actin, forward 5'-AGGCACCAGGGCGTG AT-3' and reverse 5'-GCCCACATAGGAATCCTTCTGAC-3'.

Protein extraction and western blotting. Total protein was extracted from A549 cells using the RIPA lysis buffer (Beyotime Institute of Biotechnology) according to the manufacturer's protocol. Mouse $\beta$-actin antibody (cat. no. ab8227; 1:5,000) was purchased from Abcam. Rabbit TEAD2 antibody (cat. no. PA5-40316; 1:2,000) was purchased from Thermo Fisher Scientific, Inc. Mouse CTGF (cat. no. sc-101586; 1:2,000), CYR61 (cat. no. sc-374129; 1:2,000) and Inhba (cat. no. sc-166503; 1:1,000) antibodies were purchased from Santa Cruz Biotechnology, Inc. Horseradish peroxidase-conjugated secondary antibodies anti-mouse (cat. no. ab6789; 1:10,000) 
and rabbit (cat. no. ab6721; 1:10,000) were purchased from Abcam. For western blotting, $20 \mu \mathrm{g}$ total protein was loaded in each lane after determination of the protein concentration using the bicinchoninic acid method. Proteins were separated by SDS-PAGE on $8 \%$ gels and transferred onto a PVDF membrane. The membrane was then blocked in 5\% non-fat milk at room temperature for $1 \mathrm{~h}$, followed by incubation with primary antibodies overnight at $4{ }^{\circ} \mathrm{C}$. On the following day, the membranes were incubated with the appropriate secondary antibody for $1 \mathrm{~h}$ at room temperature. The blots were developed with ECL prime western blotting detection reagents (GE Healthcare Life Sciences). Relative band intensity was quantified with ImageJ software (version. 1.8.0; National Institutes of Health).

Overexpression of TEAD2. A549 cDNA was prepared by TransScript First-Strand cDNA Synthesis SuperMix (Beijing TransGen Biotech Co., Ltd.) following the manufacturer's protocol. The open reading frame of TEAD2 was amplified from A549 cDNA and ligated into a pcDNA3.1 plasmid (Addgene, Inc.) with PrimeSTAR Max DNA Polymerase (Takara Bio, Inc.). The primers used to amplify TEAD2 were: Forward, 5'-AAGCTTATGGGGGAACCCCGGGC-3' and reverse, 5'-GAATTCTCAGTCCCTGACCAGG-3'. For the overexpression of TEAD2, $2 \mu \mathrm{g}$ pcDNA3.1-TEAD2 plasmid or empty vector was transfected into A549 cells using Lipofectamine 3000 (Invitrogen; Thermo Fisher Scientific, Inc.) according to the manufacturer's protocol; the cells were harvested for subsequent experiments after 2 days.

Dual-luciferase reporter assay. TargetScan version 7.1 software (24) was first used to predict the interaction between TEAD2 and miR-608. The 3'UTR of TEAD2 mRNA was amplified from A549 cDNA using PrimeSTAR Max DNA Polymerase and ligated into the pGL3 dual-luciferase plasmid (Promega Corporation) using the following primers: Forward, 5'-TCTAGA ACCCCA AAACTGGCTC-3' and reverse, 5'-TCTAGAATCCTCTGTCAGAAC-3'. The thermocycling conditions were $98^{\circ} \mathrm{C}$ for $10 \mathrm{sec}, 55^{\circ} \mathrm{C}$ for $15 \mathrm{sec}$ and $72^{\circ} \mathrm{C}$ for $15 \mathrm{sec}$, for 30 cycles. Point mutations were introduced into pGL3-TEAD2 3'UTR wild-type (WT; 5'-CCACCCC-3') for the construction of pGL3-TEAD2 3'UTR mutant (Mut; 5'-CGACGCC-3'). In total, $1 \times 10^{5}$ A549 cells/well were seeded in 24-well plates. miR-608 mimic $(20 \mathrm{nM})$ or miR-NC mimic $(20 \mathrm{nM})$ were cotransfected with $(0.4 \mathrm{mg})$ pGL3-TEAD2 3'UTR-WT or $(0.4 \mathrm{mg})$ pGL3-TEAD2 3'UTR-Mut into A549 cells using Lipofectamine 3000 (Invitrogen; Thermo Fisher Scientific, Inc.). After $48 \mathrm{~h}$, cells were harvested and the relative luciferase activity was determined using a Dual-Luciferase Reporter System kit (Promega Corporation) with Renilla luciferase activity as the control.

Statistical analysis. All data were analyzed using GraphPad Prism 6.0 (GraphPad Software, Inc.). Each experiment was repeated at least 3 times. Data are presented as the mean \pm SD. The differences between two groups were analyzed using Student's t-test. Multiple groups were compared using one-way ANOVA followed by Newman-Keuls post hoc test. $\mathrm{P}<0.05$ was considered to indicate a statistically significant difference.

\section{Results}

miR-608 regulates cisplatin sensitivity in A549 cells. Cisplatin inhibits cancer cell growth by inducing apoptosis (25). Furthermore, a previous study identified that miR-608 is a proapoptotic miRNA in NSCLC cells (26). To investigate whether miR-608 was involved in regulating cisplatin sensitivity in NSCLC cells, miR-608 was overexpressed, and the cytotoxic effect of cisplatin in A549 cells was subsequently detected. Compared with the miR-NC mimic group, transfection of miR-608 mimic significantly increased the level of miR-608 in A549 cells (Fig. 1A). The cell viability assay identified that there was no significant difference in viability between the miR-NC and miR-608 mimic groups (Fig. 1B). However, cisplatin $(5,10,15$ and $20 \mu \mathrm{M})$ inhibited A549 cell viability in a dose dependent manner and miR-608 overexpression increased cisplatin-induced cytotoxicity in A549 cells (Fig. 1C), suggesting that miR-608 could sensitize A549 cells to cisplatin. Additionally, transfection with miR-608 inhibitor significantly decreased the expression level of miR-608 in A549 cells (Fig. 1D). Downregulation of miR-608 showed no significant effect on cell viability (Fig. 1E). Moreover, the miR-608 inhibitor attenuated cisplatin-induced cytotoxicity (Fig. 1F).

As low concentrations $(5 \mu \mathrm{M})$ of cisplatin induced only mild inhibition of cell viability, this concentration was selected to study the function of miR-608 during the induction of cell apoptosis following cisplatin exposure. Flow cytometry analysis revealed that cisplatin treatment $(5 \mu \mathrm{M})$ induced cell apoptosis compared with the control group, and that this effect was enhanced by miR-608 overexpression (Fig. 2A and B). Collectively, the present results suggested that miR-608 is associated with cisplatin sensitivity in NSCLC cells.

miR-608 represses TEAD2 expression in A549 cells. The YAP-TEAD2 complex is pivotal for cell survival and for cancer cell stemness, promoting chemoresistance in several types of cancer (27). Interestingly, the RT-qPCR results of the current study indicated that, compared with the miR-NC mimic group, TEAD2 expression level was significantly decreased by overexpression of miR-608 in A549 cells (Fig. 3A). The western blot analysis showed that the protein expression level of TEAD2 was significantly decreased in A549 cells transfected with miR-608 mimic compared with the miR-NC mimic group (Fig. 3B and C). Moreover, inhibition of miR-608 increased TEAD2 mRNA and protein expression levels in A549 cells compared with the NC (Fig. 3D-F), suggesting that miR-608 may alter the activity of the YAP-TEAD2 complex. Furthermore, compared with the miR-NC mimic group, the mRNA expression levels of CTGF, CYR61 and Inhba, three target genes of the YAP-TEAD2 complex (28-30), were decreased in A549 cells transfected with miR-608 mimic (Fig. 4A). Additionally, the protein expression levels of CTGF, CYR61 and Inhba were decreased following overexpression of miR-608 in A549 cells (Fig. 4B and C). The present data suggested that miR-608 negatively regulates TEAD2 expression and inactivates the YAP-TEAD2 complex, which may be involved in the increased sensitivity to cisplatin mediated by miR-608 in NSCLC cells.

TEAD2 is directly targeted by miR-608 in A549 cells. To examine whether miR-608 directly regulates TEAD2 

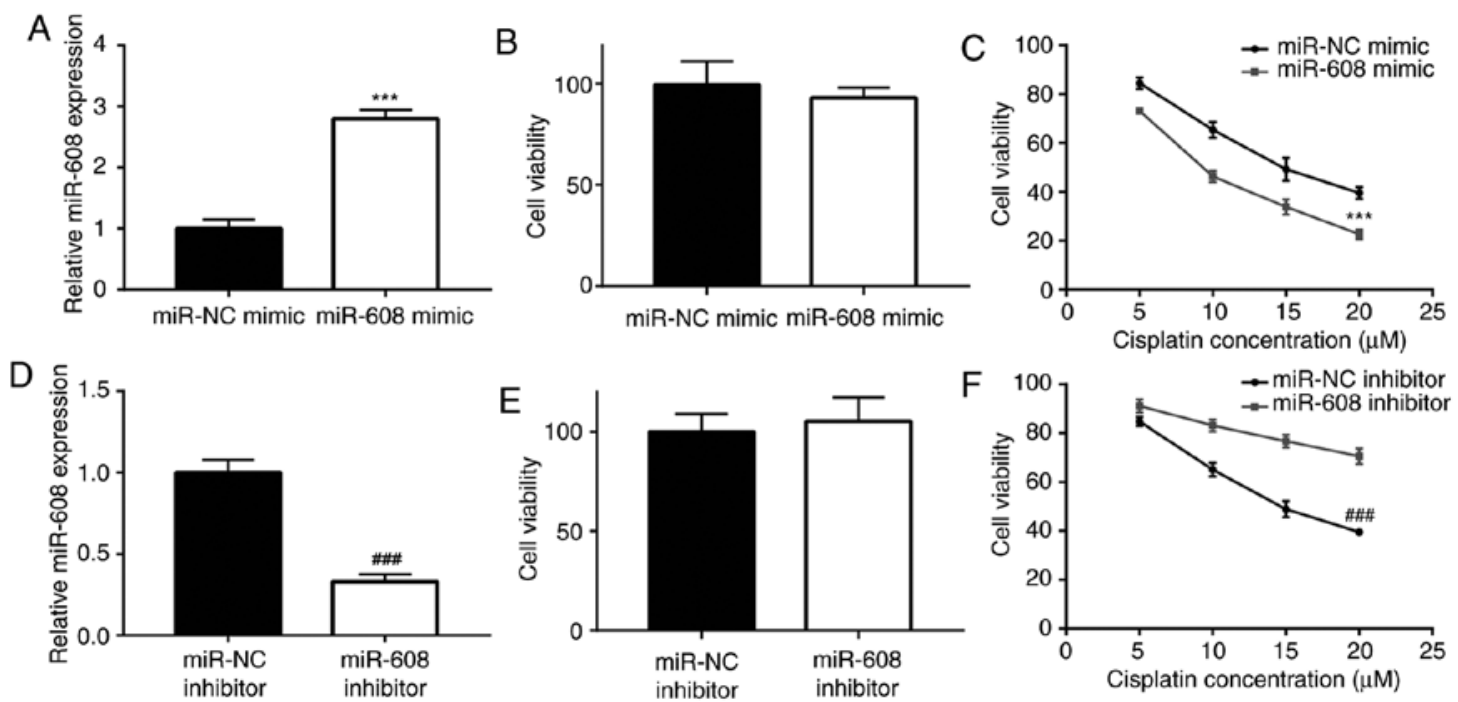

Figure 1. miR-608 positively regulates cisplatin sensitivity in non-small cell lung cancer cells. (A) Transfection of miR-608 mimic increased miR-608 expression in A549 cells. (B) Overexpression of miR-608 did not significantly affect viability of A549 cells. (C) Cisplatin treatment decreased viability of A549 cells in a dose dependent manner $(5,10,15$ and $20 \mu \mathrm{M})$. (D) Transfection of miR-608 inhibitor decreased miR-608 expression in A549 cells. (E) miR-608 inhibition did not significantly affect viability of A549 cells. (F) Cisplatin treatment decreased viability of A549 cells in a dose dependent manner (5, 10, 15 and 20 $\mu$ M). ${ }^{* * *} \mathrm{P}<0.001$ vs. miR-NC mimic; ${ }^{\# \#} \mathrm{P}<0.001$ vs. miR-NC inhibitor. miR, microRNA; NC, negative control.
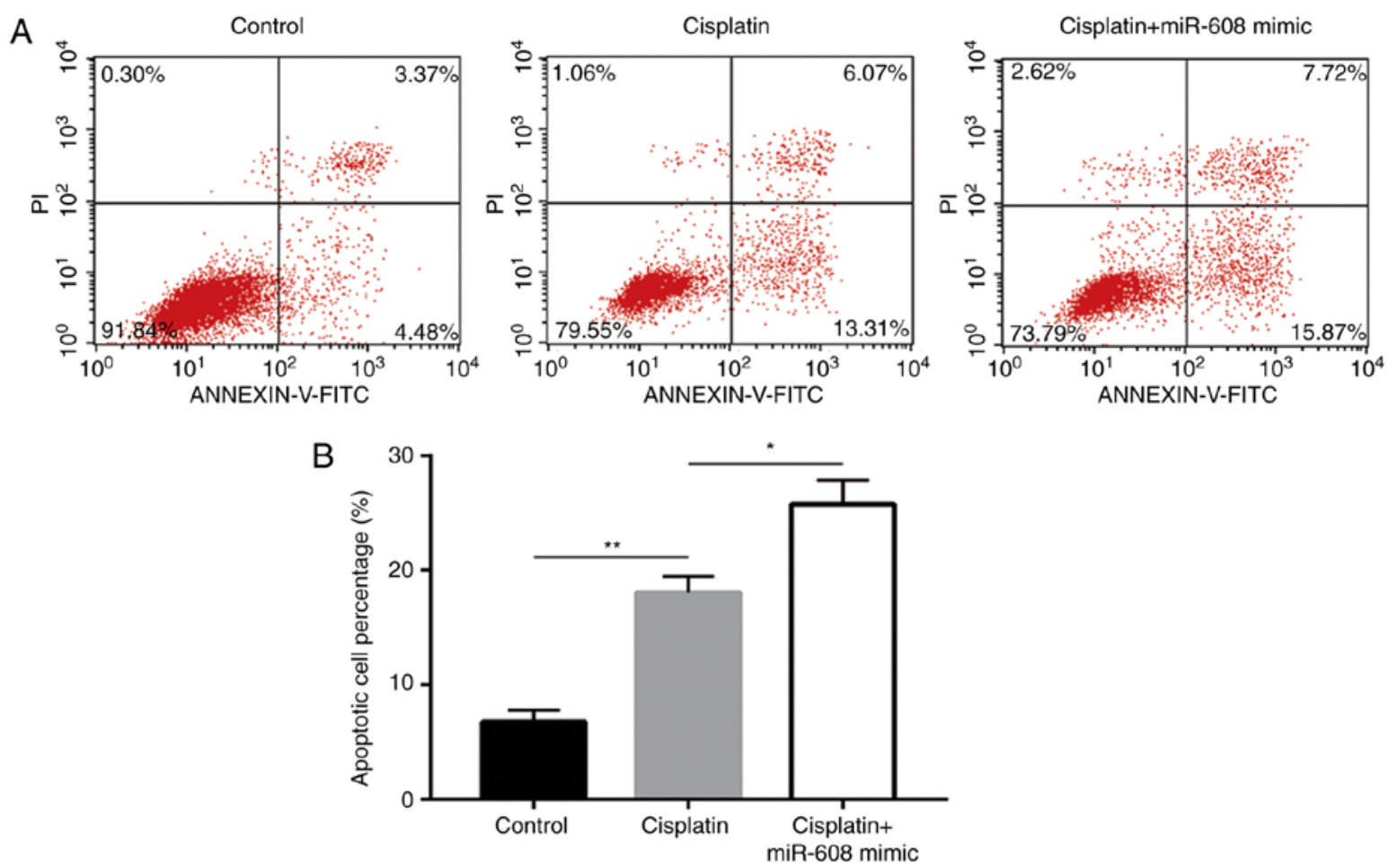

Figure 2. miR-608 increases cisplatin-induced apoptosis in non-small cell lung cancer cells. (A) Cisplatin treatment at a concentration of $5 \mu \mathrm{M}$ induced apoptosis of A549 cells, and the apoptotic rate was increased following miR-608 mimic transfection. (B) Quantification of cell apoptosis. ${ }^{*} \mathrm{P}<0.05$; ${ }^{* *} \mathrm{P}<0.01$. miR, microRNA; PI, propidium iodide.

expression in NSCLC cells, bioinformatic analysis was performed to predict the target genes of miR-608 using TargetScan software. The sequence alignment indicated that there was a putative binding site of miR-608 in the 3'UTR of TEAD2 (Fig. 5A). A luciferase assay identified that, compared with the miR-NC mimic group, the relative luciferase activity of TEAD2 3'UTR-WT was decreased following miR-608 overexpression; however, there was no significant difference in the relative luciferase activity of TEAD2 3'UTR-Mut between the miR-NC and miR-608 mimic groups (Fig. 5B). The present results suggested that miR-608 repressed TEAD2 expression by binding to its 3'UTR in NSCLC cells.

miR-608 sensitizes A549 cells to cisplatin by regulating $T E A D 2$. To examine whether TEAD2 was involved in the 
A
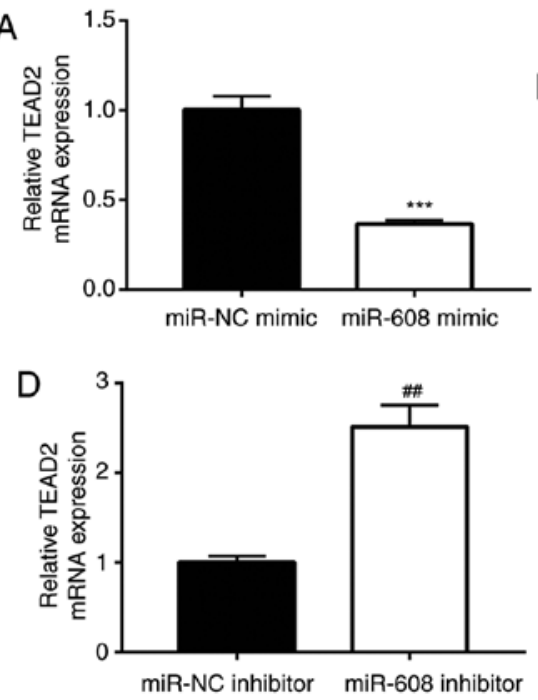

B

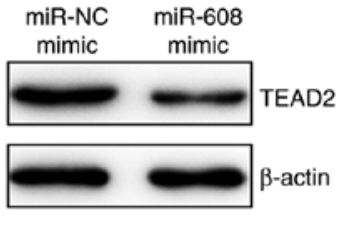

$\mathrm{E}$

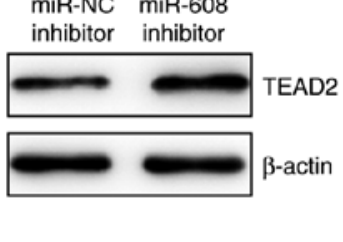



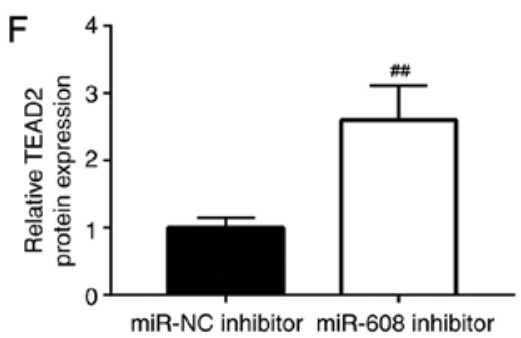

Figure 3. TEAD2 expression is repressed by miR-608 in non-small cell lung cancer cells. (A) Overexpression of miR-608 decreased the mRNA expression level of TEAD2 in A549 cells. (B) Overexpression of miR-608 decreased the protein expression level of TEAD2 in A549 cells. (C) Semi-quantification of TEAD2 protein expression levels. (D) Downregulation of miR-608 increased the mRNA expression level of TEAD2 in A549 cells. (E) Downregulation of miR-608 increased the protein expression level of TEAD2 in A549 cells. (F) Semi-quantification of TEAD2 protein expression levels. ${ }^{* *} \mathrm{P}<0.01,{ }^{* * * *} \mathrm{P}<0.001 \mathrm{vs}$. miR-NC mimic; \#" $\mathrm{P}<0.001$ vs. miR-NC inhibitor. TEAD2, TEA domain transcription factor 2; miR, microRNA; NC, negative control.

A
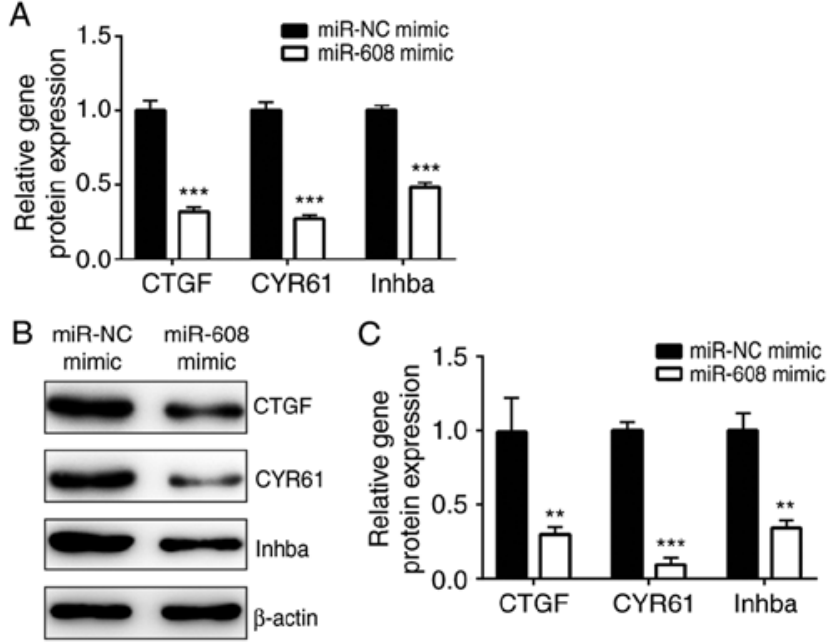

Figure 4. Overexpression of miR-608 inhibits the expression levels of genes downstream of the yes-associated protein-TEA domain transcription factor 2 complex. (A) mRNA expression levels of CTGF, CYR61 and Inhba were decreased following overexpression of miR-608 in A549 cells. (B) Protein expression levels of CTGF, CYR61 and Inhba were decreased following overexpression of miR-608 in A549 cells. (C) Semi-quantification of CTGF, CYR61 and Inhba protein expression levels. ${ }^{* *} \mathrm{P}<0.01,{ }^{* * *} \mathrm{P}<0.001$ vs. miR-NC mimic. CTGF, connective tissue growth factor; CYR61, cysteine-rich angiogenic inducer 61; Inhba, inhibin subunit $\beta \mathrm{A}$; miR, microRNA; NC, negative control.

miR-608-mediated cisplatin sensitivity in NSCLC cells, A549 cells were transfected with miR-608 mimic and pcDNA3.1-TEAD2 or empty pcDNA3.1, and the sensitivity to cisplatin was detected. Compared with A549 cells transfected with empty pcDNA3.1, transfection of pcDNA3.1-TEAD2 significantly increased the protein expression level of TEAD2 (Fig. 6A and B). Cell viability assay results suggested that miR-608-induced cisplatin

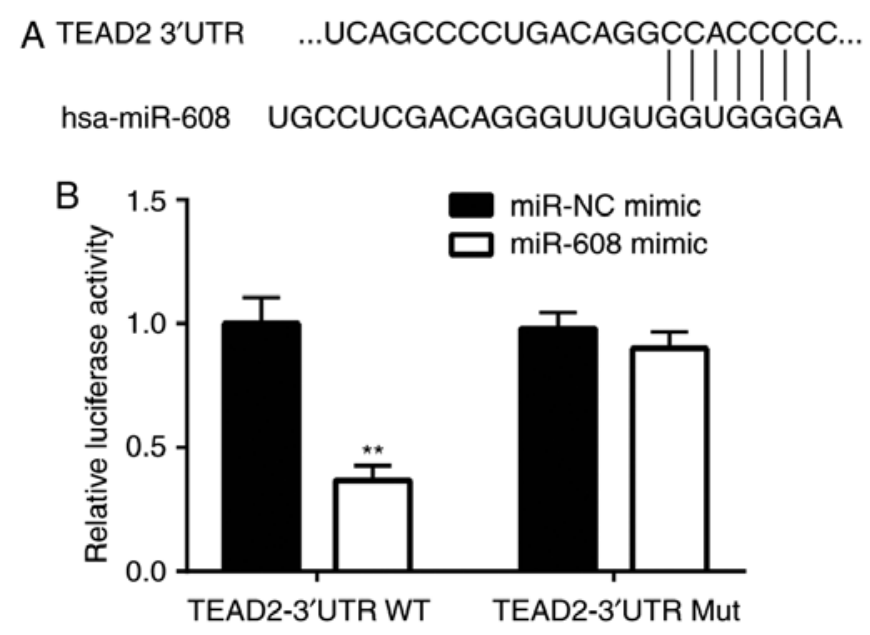

Figure 5. TEAD2 is regulated by miR-608 in non-small cell lung cancer cells. (A) Using TargetScan software, sequence alignment identified a putative binding site of miR-608 on the 3'UTR of TEAD2. (B) Relative luciferase activity of TEAD2 3'UTR-WT was significantly decreased following miR-608 overexpression in A549 cells, as assessed by dual-luciferase reporter assay. Luciferase activity of TEAD2 3'UTR-Mut was not affected following miR-608 overexpression. ${ }^{* *} \mathrm{P}<0.01$ vs. miR-NC mimic. miR, microRNA; NC, negative control; Mut, mutant; WT, wild-type; 3'UTR, 3'untranslated region; TEAD2, TEA domain transcription factor 2 .

sensitivity in A549 cells was reversed by overexpression of TEAD2 (Fig. 6C). Again, $5 \mu \mathrm{M}$ cisplatin was selected to study the role of TEAD2 during the enhancement of cisplatin-induced cell apoptosis by miR-608 overexpression. Flow cytometry analysis identified that miR-608 overexpression enhanced cisplatin-induced apoptosis in A549 cells, and this effect was attenuated by TEAD2 overexpression (Fig. 6D and E). The present results suggested that the role of miR- 608 in cisplatin sensitivity was mediated by the regulation of TEAD2 in NSCLC cells. 

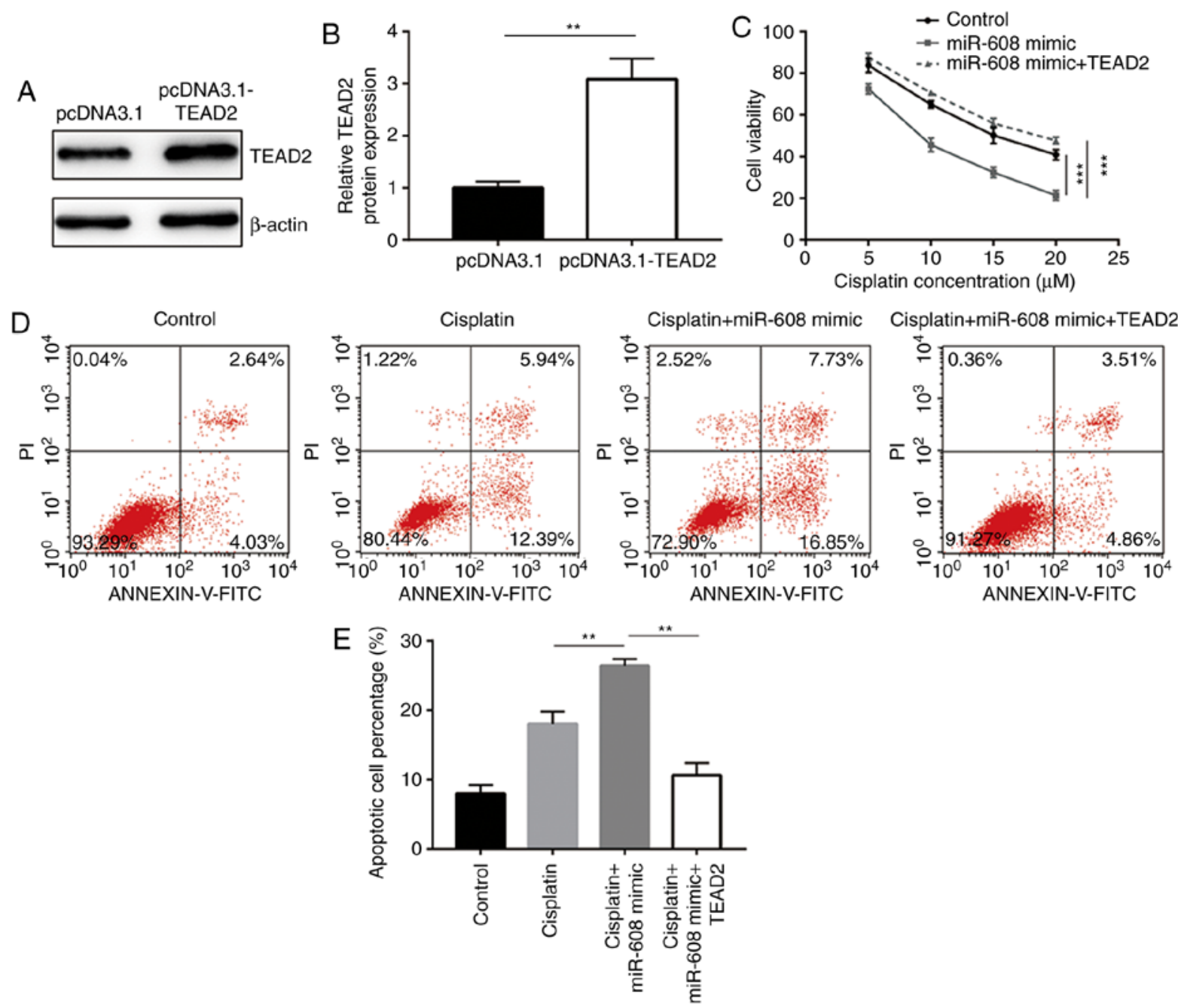

Figure 6. miR-608 mediates cisplatin sensitivity by regulating TEAD2 in non-small cell lung cancer cells. (A) Transfection of TEAD2 overexpression plasmid increased the protein expression level of TEAD2 in A549 cells. (B) Semi-quantification of the protein expression level of TEAD2. (C) Overexpression of miR-608 increased cisplatin sensitivity in A549 cells, and overexpression of TEAD2 reversed this effect. (D) Overexpression of miR-608 increased cisplatin-induced apoptosis in A549 cells, and overexpression of TEAD2 attenuated this effect. (E) Quantification of apoptotic cells. ${ }^{* *} \mathrm{P}<0.01,{ }^{* * * *} \mathrm{P}<0.001$. miR, microRNA; TEAD2, TEA domain transcription factor 2; PI, propidium iodide.

\section{Discussion}

Similarly to other chemotherapy drugs, intrinsic resistance to cisplatin represents a major limitation to its clinical efficacy (31). Although cisplatin is effective in the initial stages of treatment, chemoresistance limits its long-term efficacy, leading to mortality (4). Accumulating evidence demonstrates that dysregulation of miRNAs contributes to cisplatin resistance in cancer cells. miRNA profiling in human ovarian cancer identified that miR-125 is essential for cell survival and cisplatin resistance (32). In NSCLC, several miRNAs are reported to regulate cell apoptosis and cisplatin sensitivity by repressing various target genes $(33,34)$. Zhang et al (35) demonstrated that miR-181c targets WNT inhibitory factor 1 , a regulator of the Wnt signaling pathway, promoting the development of cisplatin resistance in NSCLC cells. miR-101, a tumor suppressor gene, is a negative regulator of Rho associated coiled-coil containing protein kinase 2 and mediates cisplatin sensitivity of NSCLC cells by regulating EMT (36). Additionally, miR-608 was identified as a proapoptotic miRNA in
NSCLC cells (26). A recent study demonstrated that the expression level of miR-608 is decreased in NSCLC tissues compared with normal tissues, and miR-608 overexpression inhibits migration and invasion of NSCLC cells (37). In the present study, miR-608 overexpression increased cisplatin sensitivity in A549 cells by increasing cisplatin-induced cell apoptosis. Conversely, downregulation of miR-608 decreased cisplatin sensitivity in A549 cells by attenuating cisplatin-induced cell apoptosis, indicating that miR-608 regulated chemotherapy sensitivity in NSCLC cells. The present data suggested that miR-608 may serve an important role in mediating cisplatin resistance in NSCLC cells.

TEAD2 is a transcription factor involved in the maintenance of cell survival (27). The activity of TEAD2 depends on the interaction with YAP, promoting cell proliferation and inhibiting cell death (19). YAP is upregulated in NSCLC and the increased activity of the YAP-TEAD2 complex is involved in the proliferative ability and chemoresistance of NSCLC cells (38). Interestingly, although YAP was identified to be regulated by several miRNAs $(39,40)$, the regulation of 
TEAD2 by miRNAs remains unclear. In the present study, miR-608 was identified to negatively regulate TEAD2 expression in A549 cells. The present bioinformatic analysis and dual-luciferase reporter assay suggested that TEAD2 is a direct target gene of miR-608 in A549 cells. Zhang et al (41) reported that the YAP-TEAD2 complex is essential for chemoresistance in NSCLC cells. In the present study, cell viability assays suggested that overexpression of TEAD2 reversed miR-608-induced increase in cisplatin sensitivity in A549 cells. Moreover, TEAD2 overexpression decreased miR-608-induced A549 cell apoptosis in the presence of cisplatin. Collectively, the present results suggested that miR-608 repressed TEAD2 expression, sensitizing A549 cells to cisplatin.

In conclusion, the present study suggested that miR-608 sensitized NSCLC cells to cisplatin by regulating TEAD2. The present finding suggested that miR-608 could be a potential therapeutic target to treat patients with NSCLC.

\section{Acknowledgements}

Not applicable.

\section{Funding}

No funding was received.

\section{Availability of data and materials}

The datasets used and/or analyzed during the current study are available from the corresponding author on reasonable request.

\section{Authors' contributions}

YW and YiG conceived and designed the present study. YW, FL, DM, YuG and RL performed the experiments. YW and FL coordinated the research and analyzed the data. YiG wrote the manuscript and supervised the study.

\section{Ethics approval and consent to participate}

Not applicable.

\section{Patient consent for publication}

Not applicable.

\section{Competing interests}

The authors declare that they have no competing interests.

\section{References}

1. Torre LA, Bray F, Siegel RL, Ferlay J, Lortet-Tieulent J and Jemal A: Global cancer statistics, 2012. CA Cancer J Clin 65 87-108, 2015.

2. Ma J, Ward EM, Smith R and Jemal A: Annual number of lung cancer deaths potentially avertable by screening in the United States. Cancer 119: 1381-1385, 2013

3. Laack E, Bokemeyer C and Hossfeld DK: Adjuvant chemotherapy after complete resection of non-small cell lung cancer Dtsch Arztebl Int 105: 249-254, 2008.
4. Chang A: Chemotherapy, chemoresistance and the changing treatment landscape for NSCLC. Lung Cancer 71: 3-10, 2011.

5. Bartel DP: MicroRNAs: Genomics, biogenesis, mechanism, and function. Cell 116: 281-297, 2004.

6. Krol J, Loedige I and Filipowicz W: The widespread regulation of microRNA biogenesis, function and decay. Nat Rev Genet 11: 597-610, 2010.

7. Iorio MV and Croce CM: MicroRNA dysregulation in cancer: Diagnostics, monitoring and therapeutics. A comprehensive review. EMBO Mol Med 4: 143-159, 2012.

8. Moriyama T, Ohuchida K, Mizumoto K, Yu J, Sato N, Nabae T, Takahata S, Toma H, Nagai E and Tanaka M: MicroRNA-21 modulates biological functions of pancreatic cancer cells including their proliferation, invasion, and chemoresistance. Mol Cancer Ther 8: 1067-1074, 2009.

9. Ceppi P, Mudduluru G, Kumarswamy R, Rapa I, Scagliotti GV, Papotti M and Allgayer $\mathrm{H}$ : Loss of miR-200c expression induces an aggressive, invasive, and chemoresistant phenotype in non-small cell lung cancer. Mol Cancer Res 8: 1207-1216, 2010.

10. Allen KE and Weiss GJ: Resistance may not be futile: MicroRNA biomarkers for chemoresistance and potential therapeutics. Mol Cancer Ther 9: 3126-3136, 2010.

11. Jin Z, Guan L, Song Y, Xiang GM, Chen SX and Gao B: MicroRNA-138 regulates chemoresistance in human non-small cell lung cancer via epithelial mesenchymal transition. Eur Rev Med Pharmacol Sci 20: 1080-1086, 2016.

12. Wu S, Yuan W, Shen Y, Lu X, Li Y, Tian T, Jiang L, Zhuang X, Wu J and Chu M: The miR-608 rs4919510 polymorphism may modify cancer susceptibility based on type. Tumour Biol 39: $1010428317703819,2017$.

13. Liu H, Zhou Y, Liu Q, Xiao G, Wang B, Li W, Ye D and Yu S: Association of miR-608 rs4919510 polymorphism and cancer risk: A meta-analysis based on 13,664 subjects. Oncotarget 8: 37023-37031, 2017.

14. Othman N, In LL, Harikrishna JA and Hasima N: Bcl-xL silencing induces alterations in hsa-miR-608 expression and subsequent cell death in A549 and SK-LU1 human lung adenocarcinoma cells. PLoS One 8: e81735, 2013.

15. Mesrouze Y, Bokhovchuk F, Meyerhofer M, Fontana P, Zimmermann C, Martin T, Delaunay C, Erdmann D, Schmelzle T and Chène P: Dissection of the interaction between the intrinsically disordered YAP protein and the transcription factor TEAD. Elife 6: e25068, 2017.

16. Zhang H, Liu CY, Zha ZY, Zhao B, Yao J, Zhao S, Xiong Y, Lei QY and Guan KL: TEAD transcription factors mediate the function of TAZ in cell growth and epithelial-mesenchymal transition. J Biol Chem 284: 13355-13362, 2009.

17. Landin-Malt A, Benhaddou A, Zider A and Flagiello D: An evolutionary, structural and functional overview of the mammalian TEAD1 and TEAD2 transcription factors. Gene 591: 292-303, 2016

18. Sawada A, Kiyonari H, Ukita K, Nishioka N, Imuta Y and Sasaki H: Redundant roles of Tead1 and Tead 2 in notochord development and the regulation of cell proliferation and survival. Mol Cell Biol 28: 3177-3189, 2008.

19. Tian W, Yu J, Tomchick DR, Pan D and Luo X: Structural and functional analysis of the YAP-binding domain of human TEAD2. Proc Natl Acad Sci USA 107: 7293-7298, 2010.

20. Lin KC, Park HW and Guan KL: Regulation of the hippo pathway transcription factor TEAD. Trends Biochem Sci 42: 862-872, 2017.

21. Lamar JM, Stern P, Liu H, Schindler JW, Jiang ZG and Hynes RO: The Hippo pathway target, YAP, promotes metastasis through its TEAD-interaction domain. Proc Natl Acad Sci USA 109: E2441-E2450, 2012.

22. Diepenbruck M, Waldmeier L, Ivanek R, Berninger P, Arnold P, van Nimwegen E and Christofori G: Tead2 expression levels control the subcellular distribution of Yap and Taz, zyxin expression and epithelial-mesenchymal transition. J Cell Sci 127: 1523-1536, 2014.

23. Livak KJ and Schmittgen TD: Analysis of relative gene expression data using real-time quantitative PCR and the 2(-Delta Delta C(T)) method. Methods 25: 402-408, 2001.

24. Agarwal V, Bell GW, Nam JW and Bartel DP: Predicting effective microRNA target sites in mammalian mRNAs. Elife 4: $\mathrm{e} 05005,2015$.

25. Bragado P, Armesilla A, Silva A and Porras A: Apoptosis by cisplatin requires p53 mediated p38alpha MAPK activation through ROS generation. Apoptosis 12: 1733-1742, 2007.

26. Othman $\mathrm{N}$ and Nagoor NH: The role of microRNAs in the regulation of apoptosis in lung cancer and its application in cancer treatment. Biomed Res Int 2014: 318030, 2014. 
27. Touil Y, Igoudjil W, Corvaisier M, Dessein AF, Vandomme J, Monté D, Stechly L, Skrypek N, Langlois C, Grard G, et al: Colon cancer cells escape 5FU chemotherapy-induced cell death by entering stemness and quiescence associated with the c-Yes/YAP axis. Clin Cancer Res 20: 837-846, 2014.

28. Di Benedetto A, Mottolese M, Sperati F, Ercolani C, Di Lauro L, Pizzuti L, Vici P, Terrenato I, Sperduti I, Shaaban AM, et al: The Hippo transducers TAZ/YAP and their target CTGF in male breast cancer. Oncotarget 7: 43188-43198, 2016.

29. Hsu PC, Tian B, Yang YL, Wang YC, Liu S, Urisman A, Yang CT, Xu Z, Jablons DM and You L: Cucurbitacin E inhibits the Yes-associated protein signaling pathway and suppresses brain metastasis of human non-small cell lung cancer in a murine model. Oncol Rep 2019 (Epub ahead of print).

30. Happé H, van der Wal AM, Leonhard WN, Kunnen SJ, Breuning $\mathrm{MH}$, de Heer E and Peters DJ: Altered Hippo signalling in polycystic kidney disease. J Pathol 224: 133-142, 2011.

31. Kelland LR, Murrer BA, Abel G, Giandomenico CM, Mistry P and Harrap KR: Ammine/amine platinum(IV) dicarboxylates: A novel class of platinum complex exhibiting selective cytotoxicity to intrinsically cisplatin-resistant human ovarian carcinoma cell lines. Cancer Res 52: 822-828, 1992.

32. Yang H, Kong W, He L, Zhao JJ, O'Donnell JD, Wang J, Wenham RM, Coppola D, Kruk PA, Nicosia SV and Cheng JQ: MicroRNA expression profiling in human ovarian cancer: MiR-214 induces cell survival and cisplatin resistance by targeting PTEN. Cancer Res 68: 425-433, 2008.

33. Bian HB, Pan X, Yang JS, Wang ZX and De W: Upregulation of microRNA-451 increases cisplatin sensitivity of non-small cell lung cancer cell line (A549). J Exp Clin Cancer Res 30: 20, 2011

34. Dong Z, Zhong Z, Yang L, Wang S and Gong Z: MicroRNA-31 inhibits cisplatin-induced apoptosis in non-small cell lung cancer cells by regulating the drug transporter ABCB9. Cancer Lett 343: $249-257,2014$.
35. Zhang $\mathrm{H}$, Hu B, Wang Z, Zhang F, Wei H and Li L: miR-181c contributes to cisplatin resistance in non-small cell lung cancer cells by targeting Wnt inhibition factor 1 . Cancer Chemother Pharmacol 80: 973-984, 2017.

36. Ye Z, Yin S, Su Z, Bai M, Zhang H, Hei Z and Cai S: Downregulation of miR-101 contributes to epithelial-mesenchymal transition in cisplatin resistance of NSCLC cells by targeting ROCK2. Oncotarget 7: 37524-37535, 2016

37. Yu HX, Wang XM, Han XD and Cao BF: MiR-608 exerts tumor suppressive function in lung adenocarcinoma by directly targeting MIF. Eur Rev Med Pharmacol Sci 22: 4908-4916, 2018.

38. Guo J, Wu Y, Yang L, Du J, Gong K, Chen W, Dai J, Li X and Xi S: Repression of YAP by NCTD disrupts NSCLC progression. Oncotarget 8: 2307-2319, 2017.

39. Liu AM, Poon RT and Luk JM: MicroRNA-375 targets Hippo-signaling effector YAP in liver cancer and inhibits tumor properties. Biochem Biophys Res Commun 394: 623-627, 2010.

40. Lei CJ, Li L, Gao X, Zhang J, Pan QY, Long HC, Chen CZ, Ren DF and Zheng G: Hsa-miR-132 inhibits proliferation of hepatic carcinoma cells by targeting YAP. Cell Biochem Funct 33: 326-333, 2015.

41. Zhang M, Zeng J, Zhao Z and Liu Z: Loss of MiR-424-3p, not miR-424-5p, confers chemoresistance through targeting YAP1 in non-small cell lung cancer. Mol Carcinog 56: 821-832, 2017.

This work is licensed under a Creative Commons Attribution-NonCommercial-NoDerivatives 4.0 International (CC BY-NC-ND 4.0) License. 